\title{
Impact of childhood exposure to a natural disaster on adult mental health: 20-year longitudinal follow-up study
}

\author{
Alexander C. McFarlane and Miranda Van Hooff
}

\section{Background}

Studies examining the impact of childhood disaster exposure on the development of adult psychopathology report increased rates of post-traumatic stress disorder (PTSD) and other psychopathology.

\section{Aims}

To examine the rates of PTSD and other lifetime DSM-IV disorders in adults exposed to an Australian bushfire disaster as children in 1983 using a matched control sample recruited at the time of the original study.

\section{Method}

A total of 1011 adults recruited from an original sample of 1531 were assessed 20 years following the fires using the Composite International Diagnostic Interview (CIDI-2.1).

\section{Results}

Results showed only a small direct impact of the fires on adult psychiatric morbidity. A higher proportion of bushfire survivors met criteria for 'any DSM-IV disorder' and 'any anxiety'.

\section{Conclusions}

Findings suggest that the long-term impact of a prominent trauma in childhood should be assessed in the context of other lifetime trauma in order to provide a more accurate account of PTSD prevalence rates.

\section{Declaration of interest}

None.
A number of longitudinal studies examine the psychological outcome of experiencing a disaster in childhood. ${ }^{1-18}$ To our knowledge only three of these studies, however, have followed the disaster-affected sample into adulthood and have included a matched control sample. Green et al, ${ }^{7}$ conducted a 17 -year follow-up of 99 survivors of the Buffalo Creek Dam collapse in 1972. Yule et $a l^{8}$ and Bolton et $a l^{9}$ conducted a 5- to 8-year follow-up of 216 survivors of the sinking of the cruise ship Jupiter in Greek waters in 1988. Finally, Morgan and colleagues ${ }^{17}$ conducted a 33-year follow-up of 41 survivors of the 1966 'Aberfan' disaster, in which a coal slagheap collapsed onto a primary school in South Wales killing 116 children. These studies reported significantly higher rates of lifetime posttraumatic stress disorder (PTSD) in disaster survivors (34-51.5\%) compared with controls (3.4-20\%). ${ }^{7,8,17}$ Current prevalence rates of PTSD in survivors ranged from 4 to $29 \% .^{7,8,17}$ Only one study reported significantly higher lifetime rates of anxiety disorder and affective disorder in disaster survivors compared with controls. ${ }^{9}$

There are two major methodological limitations to these studies. First, survivors are compared with a matched comparison group recruited at the time of follow-up rather than at the time of the original disaster, raising questions about the adequacy and matching of the comparison samples. Furthermore, not one of these studies incorporated an assessment of the role or impact of other lifetime trauma in the disaster-affected population. Given the significant rates of lifetime trauma exposure found in general population studies, including a PTSD rate of $20 \%$ from other traumas among the comparison sample in the Aberfan study, ${ }^{17}$ the contribution of additional trauma exposure needs to be taken into account in long-term studies of disaster survivors. ${ }^{19,20}$

The aim of the current study is to investigate the adult psychiatric outcomes of childhood exposure to a major Australian bushfire in the context of other lifetime trauma by utilising an unexposed control sample recruited at the time of the initial disaster.

\section{Method}

\section{Sample}

\section{The bushfire-exposed cohort}

The original bushfire-exposed cohort consisted of 806 children attending primary school in an easily demarcated region of South Australia that was vastly devastated by the Ash Wednesday Fires on 16 February 1983. Participants were recruited as children in the 2-year period following the fires, with initial findings being reported elsewhere. ${ }^{21-23}$ Of these 806 children, 440 (54.6\%) completed the full follow-up protocol and a further $540(67 \%)$ completed at least one part of the protocol. All but one of the schools attended by the children on the day of the fires were physically threatened by the bushfire, with the fire encroaching on to the school grounds. The mean age of the bushfire sample at the time of the disaster was 8.44 years (range 3.23-13.49) and at follow-up was 28.64 years (range 23-34).

\section{The control sample}

In 1985,725 primary school children from a neighbouring region in South Australia that was not directly devastated by the fires were recruited as a comparison sample. The schools attended by these children were both unaffected by the bushfire and sociodemographically matched to the schools in the fire-affected region. ${ }^{23}$ Of these 725 children, $382(52.7 \%)$ completed the full follow-up study protocol and $471(65 \%)$ completed at least one part of the protocol. The mean age of the control sample at the time of the disaster was 7.39 years (range 1.67-13.11) and at follow-up was 27.66 years (range 22-33). To ensure that socioeconomic advantage/disadvantage had not emerged between the regions in the interim 20-year period that could account for differences, the Socio-Economic Indexes for Areas (SEIFA) obtained from the Australian Bureau of Statistics (ABS) was 
compared for the control group council region and the two bushfire council regions in $2001 .^{24}$

A register of all primary school children in 1983-1985 was compiled using data from the original questionnaires and archived school admission records. Current contact information was obtained through the State Department of Births, Deaths and Marriages, the Australian Electoral Commission, and an online telephone directory. A National Death Registry was used to identify the deceased. Initial contact with the participants was by letter, followed by a telephone call. Consenting participants completed a 1-hour telephone interview and a self-report booklet.

Interviewers were trained researchers with a Bachelor of Psychology and extensive experience in telephone recruitment, interviewing and psychiatric assessment. The appropriate ethical clearance and informed consent was obtained.

\section{Instruments}

\section{Demographics}

Demographic questions were derived from the 1997 Australian National Survey of Health and Wellbeing ${ }^{25}$ and are reported using standards employed by the ABS and the Australian Institute of Health and Welfare (AIHW). Occupations were categorised using The Australian Standard Classification of Occupations (ASCO). ${ }^{26}$ The proportion of participants residing in metropolitan, rural and remote locations in Australia was determined using the Rural, Remote Metropolitan Area Classification (RRMA) that gives an index of residential remoteness based on distance to service centres as well as distance from other people.

\section{Adult psychiatric morbidity}

Lifetime and 1-month point prevalence rates of DSM-IV ${ }^{27}$ disorder were assessed using a computerised version of the fully structured, standardised and comprehensive Composite International Diagnostic Interview (CIDI). ${ }^{28}$ Disorders classified by DSM-IV were subgrouped as follows: any depressive disorder (major depression - single episode, major depression - recurrent, depressive disorder not otherwise specified and dysthymia); any anxiety disorder (panic disorder, panic with agoraphobia, agoraphobia, specific phobia - animal, specific phobia - environmental, specific phobia - blood, specific phobia - situational, specific phobia - other, social phobia, obsessive-compulsive disorder, generalised anxiety disorder, anxiety not otherwise specified, and PTSD - based on worst event); and any eating disorder (anorexia nervosa, bulimia nervosa and eating disorder not otherwise specified). Current and lifetime DSM-IV PTSD was examined in all participants in relation to the event rated subjectively by the participant to be their 'worst lifetime traumatic event'. This event was chosen by the participant from a standard list of 10 Criterion-A events from the CIDI. ${ }^{28}$ These were direct combat, life-threatening accident, fire, flood or natural disaster, witnessed someone being badly injured or killed, rape, sexual molestation, serious physical attack or assault, threatened with a weapon/held captive/kidnapped, tortured or victim of terrorists, and any other stressful event. In addition, the researchers included seven other event types (domestic violence, witnessed domestic violence, threatened/harassed without a weapon, finding a dead body, witnessing someone die by suicide or attempt suicide, child physical abuse, child emotional abuse). These events were based on a systematic recoding of the 'other' trauma category in a random community sample. ${ }^{29}$ Participants were asked whether they had experienced any of these events and whether they had ever experienced a great shock as a result of any of these events happening to someone close to them. They were then asked to nominate which of these events was their worst lifetime event.
Post-traumatic stress disorder was assessed in reference to this self-nominated worst lifetime event. This methodology is in contrast to previous longitudinal studies on disaster-affected populations, which predominantly focus on the disaster in the exposed population and the worst lifetime traumatic event in the controls. The purpose of assessing PTSD in this way was to determine how the disaster was subjectively rated in relation to other traumatic life events that the person had experienced and whether the experience of other events has an impact on the prevalence of PTSD in relation to the bushfire.

\section{Lifetime and current prevalence of bushfire-related PTSD}

In addition to the assessment of PTSD in relation to the participant's worst lifetime event, the CIDI PTSD section was also completed for the second and third worst events to allow a more detailed examination of the prevalence of lifetime and current DSM-IV PTSD. This method also allowed an assessment of the bushfire-related PTSD, particularly how the participants rank the bushfire in the hierarchy of their stressful experiences. If the participants nominated the bushfire as their worst event, they were included in Fire Group 1. If the bushfire-exposed group did not nominate the bushfire as their worst lifetime event, but instead nominated another event as their worst, PTSD symptom questions were first asked in specific relation to this other event (Fire Group 2). This group represents individuals with PTSD symptoms related to some other worst event such as a motor vehicle accident in the group who had been exposed to the fires. Furthermore, Fire Group 3 represents all bushfire-related cases independent of whether this was rated as either their worst, second or third worst lifetime event. Therefore, Group 3 included all those in Fire Group 1 where the disaster had been their worst experience and also those in Fire Group 2 who nominated the bushfire as their second or third worst lifetime event. In total, therefore, $352(67 \%)$ of those originally exposed to the bushfires were questioned as to whether they had experienced PTSD symptoms as a direct result of the bushfire at some point in their lives. Bushfire-exposed participants were subgrouped in this way to maximise sample numbers for analysis of bushfire-related PTSD.

\section{Current self-reported levels of PTSD-related distress}

Current distress associated with PTSD symptoms was examined at 20-year follow-up using the self-report Impact of Events Scale-Revised (IES-R). ${ }^{30}$ Symptom questions were anchored to the bushfire in the survivor group and their most stressful self-nominated life event in the controls. Scores were also added to produce an overall total score and three symptom scores: intrusion, avoidance and hyperarousal.

\section{Alcohol consumption and problem drinking}

This was examined using the AUDIT (Alcohol Use Disorders Identification Test). ${ }^{31}$ This instrument consists of 10 questions to examine the quantity and frequency of alcohol consumption, drinking behaviour and the reactions or problems related to alcohol (score range $0-36$ ). The version we used has been slightly modified for the Australian context by the Centre for Drug and Alcohol Studies. Scores of 7-12 indicated harmful or hazardous drinking, and a score of 13 or greater indicated possible alcohol dependence.

\section{Analysis}

Log linear analyses $\left(\chi^{2}\right.$-tests) and independent sample $t$-tests were performed on selected baseline data to investigate differences between the responders and non-responders. The strength of these 
associations was calculated using the phi coefficient (for $\chi^{2}$ tests) and effect sizes (for $t$-tests). Primary unadjusted analysis of the effects of childhood exposure to the bushfire on demographics, alcohol consumption, and lifetime and current DSM-IV disorder was performed using chi-square analyses (for categorical variables) and $t$-tests or linear regression (for continuous variables). To account for the possible confounding effects of age and gender (known to be strong predictors of adult psychopathology), adjusted $P$-values were calculated using generalised estimating equations for demographics and lifetime psychopathology. In cases where the dependent variable was binary, generalised estimating equations were logistic regressions. For skewed sampling distributions (IES-R), the differences in medians were calculated using non-parametric tests of significance. To obtain relative risks, a binomial generalised estimating equation was used with log link, adjusting for gender and age. Analyses were performed using Statistical Analysis Systems (SAS) (version 9.1) for Windows.

\section{Results}

\section{Response rates}

Table 1 shows the response and completion rates for the entire sample. Of the original sample, $714(88.6 \%)$ of the bushfireexposed group and $624(86.1 \%)$ of the non-exposed group were relocated around the 20-year anniversary of the fires. Of those who were contacted, $440(61.62 \%)$ of the bushfire-exposed group and $382(61.21 \%)$ of the non-exposed group completed the entire protocol, and 529 bushfire-exposed and 464 control participants were interviewed using the CIDI.

\section{Responders $\boldsymbol{v}$. non-responders}

A comparison of the 1011 responders and the 520 non-responders was carried out using demographic data collected during the initial phases of the study. Overall, there were few differences between responders and non-responders on a number of variables, with non-responders more likely to be male $\left(\chi^{2}(1,1531)=4.05\right.$, $P<0.05)$ and to be younger in age $(t(1529)=-2.28, P<0.05)$. The strength of these relationships however was weak (phi coefficient: gender 0.05; effect sizes: age -0.12 ).

\section{Demographic characteristics of the follow-up sample}

Online Table DS1 reports demographic results adjusted for age at follow-up. Demographic differences between the fire-exposed and control participants suggest that exposure to the bushfire may have made the exposed group less likely to extend their education and their careers, and to be less socially mobile. Overall, a significantly greater proportion of bushfire-exposed individuals compared with controls had children $\left(\chi^{2}(1,838)=4.64\right.$, $P<0.05)$, lived in rural areas $\left(\chi^{2}(1,838)=8.38, P<0.01\right)$ and were employed as production workers $\left(\chi^{2}(1,838)=6.66, P<0.01\right)$. In contrast, a higher proportion of the control participants had completed high-school education $\left(\chi^{2}(1,838)=9.56, \quad P<0.01\right)$ and were currently employed in associate professional roles $\left(\chi^{2}(1,838)=5.43, \quad P<0.05\right)$. These differences could not be explained by differences in the advantage/disadvantage index (SEIFA) that may have emerged since the disaster between the control and fire-affected regions as measured in $2001(F=2.68$, d.f. $=2, P=0.08)$. In the earlier phase of this study, it was noted that the educational performance of the symptomatic group of exposed children significantly declined in the aftermath of the fire. ${ }^{23}$ Hence, the psychopathological measures of outcome alone do not entirely represent the disadvantage experienced by this disaster-affected group.

Overall, participants in the bushfire-exposed group were slightly older at follow-up, as a result of the fact that the comparison sample was recruited 1 year later than the bushfire-exposed sample. Hence, this is an artefact of the initial study design and has been explained elsewhere. ${ }^{23}$

\section{Lifetime and current psychopathology}

In total, $36.7 \%$ of the bushfire-exposed participants and $31.7 \%$ of the controls met criteria for any DSM-IV psychiatric disorder during their lifetime, and $15.2 \%$ of the bushfire-exposed group and $11 \%$ of controls met criteria for a disorder in the past month (Table 2). Only lifetime rates of 'any anxiety, excluding PTSD' $\left(\chi^{2}(1,993)=5.39, P=0.01\right)$ were significantly more prevalent in the bushfire-exposed group than in the controls. A higher proportion of bushfire-exposed participants compared with controls met criteria for 'any disorder' $\left(\chi^{2}(1,993)=4.32, P<0.05\right)$ and 'any anxiety' $\left(\chi^{2}(1,993)=5.66, P<0.05\right)$ in the past month. When the individual DSM-IV diagnoses were examined, the only disorder that was more prevalent in the disaster-exposed group was a lifetime history of specific phobia (environmental type) (relative risk $(\mathrm{RR})=1.92,95 \%$ CI $1.11-3.34, P=0.02$ ), whereas current environmental phobia only approached significance ( RR $=2.27,95 \%$ CI $0.97-7.62, P=0.06$ ).

Using the standard CIDI method of nominating the worst lifetime event, there were no significant differences in the rates of lifetime or 1-month prevalence of PTSD between the bushfire-exposed group and the controls. For the bushfireexposed individuals, the event most commonly nominated as their

\begin{tabular}{|c|c|c|c|c|c|c|}
\hline & \multicolumn{2}{|c|}{ Total } & \multicolumn{2}{|c|}{ Bushfire group } & \multicolumn{2}{|c|}{ Control group } \\
\hline & $n$ & $\%$ & $n$ & $\%$ & $n$ & $\%$ \\
\hline Initially recruited & 1531 & 100.0 & 806 & 100.0 & 725 & 100.0 \\
\hline \multicolumn{7}{|l|}{ Follow-up data } \\
\hline Any data & 1011 & 66.0 & 540 & 67.0 & 471 & 65.0 \\
\hline All data & 822 & 53.7 & 440 & 54.6 & 382 & 52.7 \\
\hline Booklet data & 838 & 54.7 & 450 & 55.8 & 388 & 53.5 \\
\hline Interview data (any) & 996 & 65.1 & 530 & 65.8 & 466 & 64.3 \\
\hline Composite International Diagnostic Interview data & 993 & 64.9 & 529 & 65.6 & 464 & 64.0 \\
\hline \multicolumn{7}{|l|}{ Contacted but no follow-up } \\
\hline Deceased & 20 & 1.3 & 14 & 1.7 & 6 & 0.8 \\
\hline Withdrawn & 111 & 7.3 & 60 & 7.4 & 51 & 7.0 \\
\hline Not interested & 148 & 9.7 & 77 & 9.6 & 71 & 9.8 \\
\hline Other & 48 & 3.1 & 23 & 2.9 & 25 & 3.4 \\
\hline Not contacted & 193 & 12.6 & 92 & 11.4 & 101 & 13.9 \\
\hline
\end{tabular}


Table 2 Lifetime and point (1-month) prevalence rates for DSM-IV disorders in bushfire survivors and controls ${ }^{\mathrm{a}}$

\begin{tabular}{|c|c|c|c|c|c|c|c|c|}
\hline \multirow[b]{3}{*}{ Adult symptoms } & \multicolumn{4}{|c|}{ Lifetime } & \multicolumn{4}{|c|}{ Point (1-month) } \\
\hline & $\begin{array}{l}\text { Bushfire group } \\
\quad(n=529)\end{array}$ & $\begin{array}{l}\text { Control group } \\
\qquad(n=464)\end{array}$ & & & $\begin{array}{l}\text { Bushfire group } \\
\quad(n=529)\end{array}$ & $\begin{array}{l}\text { Control group } \\
\qquad(n=464)\end{array}$ & & \\
\hline & $n(\%)$ & $n(\%)$ & $\mathrm{RR}(95 \% \mathrm{Cl})$ & $P$ & $n(\%)$ & $n(\%)$ & $\mathrm{RR}(95 \% \mathrm{Cl})$ & $P$ \\
\hline PTSD (worst event) & $41(7.8)$ & $27(5.8)$ & $1.36(0.84-2.19)$ & 0.21 & $17(3.2)$ & $11(2.4)$ & $1.35(0.63-2.90)$ & 0.44 \\
\hline Any DSM-IV disorder & $194(36.7)$ & $147(31.7)$ & $1.14(0.96-1.36)$ & 0.14 & $80(15.2)$ & $51(11.0)$ & $1.42(1.02-1.98)$ & 0.04 \\
\hline Any depressive disorder & $127(24.0)$ & $99(21.3)$ & $1.11(0.88-1.40)$ & 0.38 & $24(4.5)$ & $20(4.3)$ & $1.00(0.55-1.81)$ & 1.00 \\
\hline Any anxiety disorder, excluding PTSD & $114(21.5)$ & $74(16.0)$ & $1.37(1.05-1.78)$ & 0.02 & $57(10.8)$ & $32(6.9)$ & $1.66(1.09-2.53)$ & 0.02 \\
\hline Any eating disorder & $5(0.9)$ & $6(1.3)$ & $0.81(0.24-2.70)$ & 0.73 & $5(0.9)$ & $6(1.3)$ & $0.81(0.24-2.70)$ & 0.73 \\
\hline
\end{tabular}

worst lifetime event was a 'natural disaster' (31.6\%), suggesting the enduring impact of the bushfire, followed by 'having a family or friend in a life-threatening accident' (12.5\%) and 'witnessing someone being badly injured or killed' $(10.2 \%)$. The event most frequently nominated as the worst in the control group was 'being in a life-threatening accident' (11.2\%) followed by 'witnessing someone being badly injured or killed' $(8.6 \%)$ and 'having a family or friend in a life threatening accident' $(8.2 \%)$.

\section{Lifetime and current PTSD in relation to the bushfire}

Using the more thorough method of assessing PTSD from the three worst traumatic experiences, 352 (67\%) of the 529 bushfire survivors nominated the bushfire as one of their three worst lifetime events: $30.4 \%(n=161)$ as their worst event, $21.4 \%$ $(n=113)$ as their second worst event and $14.7 \%(n=78)$ as their third worst lifetime event. As previously reported, there were no significant differences between the bushfire group and the controls in the prevalence of PTSD resulting from the self-nominated worst lifetime event (Table 3). Interestingly, bushfire-exposed participants who nominated the bushfire as their worst lifetime event were at a lower risk of developing lifetime and current PTSD from the bushfire than the bushfire-exposed individuals who nominated some other event as their worst event (Table 4). In addition, bushfire-exposed participants who nominated the fire as one of their worst three events were less likely to develop PTSD from the fire than were the controls in relation to their self-nominated worst lifetime event. Importantly, of the 161 bushfire-exposed individuals who nominated the bushfire as their worst event, $62.7 \%(n=101)$ had reported the bushfire as their only lifetime event. The percentage in both the bushfire-exposed and the control population who had been exposed to only one event was similar being $21 \%(n=113)$ and $23 \%(n=107)$ respectively.
Interestingly, a further $114(24.5 \%)$ participants in the control group reported not being exposed to a single traumatic event during their lifetime. Therefore those in the disaster-exposed group who nominated the fire as their worst event appeared to have a lower total lifetime exposure to traumatic stress, explaining the apparent paradox of their lower rates of PTSD. This contrasts to the bushfire-exposed group who nominated another event as their worst event and who had a greater risk of PTSD (Table 4) than the controls. This group appeared to have been sensitised to subsequent trauma exposure by their experience of the bushfire.

Table 3 demonstrates that the $\mathrm{C}$ criteria (avoidance) and the $\mathrm{F}$ criterion (distress) were the diagnostic criteria that were those least frequently endorsed, and hence were the 'gate-keepers' for the diagnosis of PTSD. This effect was most pronounced in the bushfire-exposed group in relation to the nominated trauma of the fire, where only $3.1 \%$ satisfied the C (avoidance and estrangement) criteria, in contrast to the worst trauma where this was met by $17 \%$ of the sample.

\section{Current bushfire-related distress}

Twenty-seven per cent of the bushfire-exposed group reported no current bushfire-related distress (IES-R $=0$ ) at the follow-up assessment. In general, total scores on the IES-R were low, with $80 \%$ of the bushfire-exposed participants scoring 10 or below. Intrusion symptoms were most common affecting $66 \%$ of the population. Hyperarousal symptoms affected only $28 \%$ of the sample. Of those who reported some distress, the two most commonly endorsed symptoms were 'any reminders brought back feelings about it' (54.2\%) and 'pictures popped into my mind' (46.4\%).

The total IES-R scores were significantly lower in the bushfireexposed individuals (in relation to the bushfire) than in the

\begin{tabular}{|c|c|c|c|c|c|c|}
\hline \multirow[b]{2}{*}{ DSM-IV PTSD criteria } & \multicolumn{2}{|c|}{ Bushfire survivors $^{\text {a }}(n=352)$} & \multicolumn{2}{|c|}{ Bushfire survivors $^{\mathrm{b}}(n=529)$} & \multicolumn{2}{|c|}{ Control participants $^{\mathrm{b}}(n=464)$} \\
\hline & $n$ & $\%$ & $n$ & $\%$ & $n$ & $\%$ \\
\hline A2: Fear, helplessness or horror & 218 & 61.9 & 405 & 76.6 & 285 & 61.4 \\
\hline B: Re-experiencing & 118 & 33.5 & 303 & 57.3 & 236 & 50.9 \\
\hline C: Avoidance & 11 & 3.1 & 90 & 17.0 & 68 & 14.7 \\
\hline D: Hyperarousal & 64 & 18.2 & 203 & 38.4 & 161 & 34.7 \\
\hline E: Duration more than 1 month & 56 & 15.9 & 205 & 38.8 & 161 & 34.7 \\
\hline F: Significant distress or impairment & 13 & 3.7 & 77 & 14.6 & 49 & 10.6 \\
\hline PTSD lifetime & 6 & 1.7 & 41 & 7.8 & 27 & 5.8 \\
\hline PTSD current & 0 & 0 & 17 & 3.2 & 11 & 2.4 \\
\hline
\end{tabular}




\begin{tabular}{|c|c|c|c|c|c|c|c|c|}
\hline \multirow[b]{2}{*}{ Contrasts performed } & \multicolumn{4}{|c|}{ Lifetime PTSD } & \multicolumn{4}{|c|}{ Point prevalence (1-month PTSD) } \\
\hline & RR & Adjusted RR & $95 \% \mathrm{Cl}$ & $P$ & RR & Adjusted RR & $95 \% \mathrm{Cl}$ & $P$ \\
\hline \multicolumn{9}{|l|}{ Model 1} \\
\hline Fire group $1^{\mathrm{a}} v$. fire group $2^{\mathrm{b}}$ & 0.248 & 0.229 & $0.08-0.63$ & 0.004 & 0.143 & 0.133 & $0.02-0.99$ & 0.049 \\
\hline Fire group $1^{\mathrm{a}} v$. controls ${ }^{\mathrm{c}}$ & 0.428 & 0.413 & $0.15-1.17$ & 0.095 & 0.263 & 0.248 & $0.03-1.92$ & 0.181 \\
\hline Fire group $2^{b} v$. controls ${ }^{c}$ & 1.725 & 1.804 & $1.11-2.93$ & 0.017 & 1.831 & 1.869 & $0.86-4.05$ & 0.113 \\
\hline Fire group $1^{\mathrm{a}}$ and $2^{\mathrm{b}} v$. controls ${ }^{\mathrm{c}}$ & 0.859 & 0.863 & $0.46-1.62$ & 0.648 & 0.693 & 0.681 & $0.21-2.20$ & 0.521 \\
\hline \multicolumn{9}{|l|}{ Model 2} \\
\hline Fire group $3^{d} v$. controls ${ }^{c}$ & 0.294 & 0.297 & $0.12-0.72$ & 0.008 & 0.361 & 0.349 & $0.10-0.27$ & 0.011 \\
\hline
\end{tabular}

non-disaster-exposed controls (in relation to their self-nominated 'most stressful and upsetting event life') (total IES-R score: bushfire group mean $=6.92$ (s.d. $=11.0)$; control group mean $=10.08$ (s.d. $=14.0), z=3.03, P=0.002)$ with similarly significant differences existing on the avoidance and hyperarousal subscales but not the intrusion subscales. In the control sample, death of a family member or friend was the event most commonly reported as being most stressful (15.8\%). Only $8.3 \%$ of the unexposed population reported never having experienced a stressful life event.

\section{Alcohol consumption and problem drinking}

There were no significant differences in the total AUDIT score between the bushfire-exposed group and controls, or in the proportion of participants classified as 'harmful or hazardous drinkers' (30.8\% of bushfire-exposed individuals compared with $31.5 \%$ of controls $\left.\left(\chi^{2}(1,806)=0.46, P=0.831\right)\right)$ or 'alcohol dependent' $(14.2 \%$ of bushfire-exposed group compared with $10.9 \%$ of controls $\left.\left(\chi^{2}(1,806)=1.95, P=0.162\right)\right)$.

\section{Discussion}

This study is the first to follow-up a large cohort of primary school children exposed to a natural disaster, using a matched control sample recruited as children at the time of the original study, as well as being the largest long-term longitudinal study to follow a disaster-exposed cohort from childhood. Overall, the increased rates of lifetime and current 'any anxiety disorder' and 'any disorder' in the bushfire-exposed participants were relatively small, despite the fact that the bushfire-exposed children were rated as more symptomatic than the controls by their parents following the fires. ${ }^{23}$ The enduring disadvantage of the disaster was also apparent in the educational and occupational disadvantage of the bushfire-exposed group (online Table DS1). However, a caveat of this later finding is that we did not statistically control for the individual socioeconomic status of each child at the time of the disaster as the comparison group was recruited by matching indices of regional social advantage. In summary, the disaster appears to have had only a small long-term effect on anxiety rather than depressive disorders, despite the major losses of this community. ${ }^{32}$

These findings contrast to the higher rates of depression and anxiety following the long-term studies of the Aberfan ${ }^{17}$ and Jupiter disasters. ${ }^{9}$ Interestingly, the current prevalence of anxiety and depressive disorders in the bushfire-exposed participants and controls were similar to the Australian community. ${ }^{33}$ The similar prevalence rates in the exposed and control groups are likely to be as a result of the important effect of traumatic events, other than disasters in the general community, as was the case in both groups. The effect of non-disaster-related trauma has not been addressed in these previous follow-up studies.

There were no significant differences in the lifetime or 1-month prevalence of PTSD between bushfire survivors and the unexposed controls in relation to their self-nominated worst event, implying a degree of consistency in both groups with respect to how they respond to trauma over time. The event most frequently nominated by bushfire-exposed individuals as the worst event was a 'natural disaster' (31.6\%), but the remainder nominated another traumatic event, many relating to motor vehicle accidents, which had generally occurred at an older age. The association between multiple exposure to traumatic events and increased prevalence and severity of PTSD, suggesting a cumulative risk model arising from multiple trauma exposures, has been well validated in the literature. $^{34,35}$ This effect was apparent in the bushfire-exposed participants who nominated an event other than the bushfire as their worst lifetime event, with this group having a greater risk of PTSD than the controls. This finding highlights the importance of assessing other traumatic exposures in the long-term follow-up of disaster-affected populations to get true prevalence estimates of trauma-related psychopathology.

Seventy-five per cent of the bushfire-exposed group still reported some degree of distress in relation to the bushfires 20 years on. These distressing intrusions, measured by the IES-R, which have been found to drive the hyperarousal and avoidance PTSD symptoms in a group of emergency firefighters exposed to the same disaster, ${ }^{36}$ may be indicative of an ongoing risk of dysregulated affect and arousal. However, despite this distress, low rates of PTSD in relation to the bushfire were observed in the bushfire-exposed participants, although the rates of intrusive and hyperarousal symptoms were substantial (Table 3), indicative of subsyndromal PTSD. Only six of the bushfire survivors $(1.7 \%$ of those questioned in relation to the bushfire, $1.1 \%$ of the entire sample) met full diagnostic criteria for lifetime PTSD in relation to the fires. This is in stark contrast to other longitudinal studies, which have reported lifetime rates of PTSD in disaster survivors that have ranged from 34.0 to $51.5 \%{ }^{7,8,17}$ The severity of the loss of life in the Aberfan and the Buffalo Creek disaster may explain these differences but this was not the case in the Jupiter sinking where four lives were lost.

A methodological strength in this study is the use of a matched comparison group collected at the time of initial recruitment because the significant differences emerging in other studies may be as a result of the bias related to the retrospective nature of the recruitment of the control populations. Furthermore, the failure of previous studies to adequately assess non-disaster 
trauma exposure in the exposed and control groups may have led to underestimation of PTSD. Therefore future studies should explicitly assess PTSD in the disaster-exposed group in relation to both a self-nominated worst lifetime event and the disaster itself, in order to gain a more accurate representation of the true effects of the disaster in the context of other lifetime trauma.

A series of other methodological issues require consideration in the interpretation of these results. First, clinician administered measures, such as those used in the previous studies of disaster victims, have more potential for interviewer bias than the CIDI that was used in this study. ${ }^{37}$ This potential arises as a consequence of the flexibility for clinician interpretation, which can lead to bias towards the hypothesised effect, similar to the substantial bias found in unmasked clinician-rated assessments in treatment trials. ${ }^{38,39}$ As a consequence, the previous studies may have had more potential for bias in rating PTSD symptoms leading to finding the presumed association with the disaster. Second, the higher rates of PTSD in the Aberfan study may have been partly as a result of the use of ICD- $10^{40}$ criteria, whereas the current study used DSM-IV criteria. The 12-month PTSD prevalence rates using the ICD-10 criteria have been found to be double those obtained with DSM-IV criteria. ${ }^{41,42}$

Third, in contrast to the Aberfan and Buffalo Creek follow-up studies, the response bias issues in recruiting the sample were significantly less in our study due to the interview of $65 \%$ of the original sample. A limitation of the current paper arises from dealing with disaster exposure as being present or absent rather that looking at the gradient of exposure, an issue that will be addressed in further papers. Also, although the finding of social and economic disadvantage in the disaster-affected group was consistent with the progressive educational underachievement that emerged in the 2 years following the disaster, future research should control for parental education and occupation as well as the child's IQ at the time of the disaster. Finally, compensation did not play a significant role ${ }^{41}$ in the response of our sample. In contrast, the Buffalo Creek disaster and the Jupiter samples were recruited in the course of their legal action against the defendants. ${ }^{43}$

In summary, this study provides some support for long-term effects of childhood disaster on those exposed. Importantly, however, the effects are much more subtle than has previously been reported and should be viewed through the lens of the significant impact of other lifetime traumatic events. The lack of differences between the disaster-exposed group and the comparison group do not appear to be solely a result of the disaster having little effect on the disaster-exposed group, but because of the overriding impact of other trauma in both the control group and the survivors, and the unique methodology employed in the current study to document this.

Alexander C. McFarlane, MB BS (Hons), MD, FRANZCP, Dip Psychother, Miranda Van Hooff, BA (Hons), The Centre for Military and Veterans' Health, Miranda Van Hooff, BA (Hons), The Centre for
University of Adelaide, South Australia, Australia

Correspondence: Miranda Van Hooff, Centre for Military and Veterans' Health, Level 2/122 Frome Street, Adelaide, South Australia 5000, Australia. Email: miranda.vanhooff@adelaide.edu.au

First received 1 May 2008, final revision 25 Jan 2009, accepted 10 Feb 2009

\section{Funding}

This research was supported by a project grant from the Australian National Health and Medical Research Council (NHMRC Project Grant ID 201813 and program grant 300403).

\section{Acknowledgements}

The authors also wish to acknowledge the following organisations and personnel for their assistance and support with the project: Nancy Briggs from the University of Adelaide Data Management and Analysis Centre, the Births Deaths and Marriages Registration Office of South Australia, the Australian Institute of Health and Welfare, the Australian Electora South Australia, the Australan institur Commission and the teachers, principals and staff from the 8 schools that participated in the study. A special thanks to all participants who generously gave their time, support and cooperation with the project.

\section{References}

1 Goenjian AK, Pynoos RS, Steinberg A, Najarian LM, Asarnow J, Karayan I, et al. Psychiatric comorbidity in children after the 1988 earthquake in Armenia. J Am Acad Child Adolesc Psychiatry 1995; 34: 1174-84.

2 Hsu C-C, Choong M-Y, Yang P, Yen C-F. Posttraumatic stress disorder among adolescent earthquake victims in Taiwan. J Am Acad Child Adolesc Psychiatry 2002; 4: 875-81.

3 Goenjian AK, Molina L, Steinberg AM, Fairbanks LA, Alvarez ML, Goenjian HA, et al. Posttraumatic stress and depressive reactions among Nicaraguan adolescents after hurricane Mitch. Am J Psychiatry 2001; 158: 788-94.

4 La Greca AM, Silverman WK, Wasserstein SB. Children's predisaster functioning as a predictor of posttraumatic stress following hurricane Andrew. J Consult Clin Psychol 1998; 66: 883-92.

5 Shaw JA, Applegate B, Schorr C. Twenty-one month follow-up study of school-age children exposed to hurricane Andrew. J Am Acad Child Adolesc Psychiatry 1996; 35: 359-64.

6 Dollinger SJ. Lightning-strike disaster among children. Br J Med Psychol 1985; 58: $375-83$

7 Green BL, Grace M, Vary MG, Kramer TL, Gleser GC, Leonard AC. Children of disaster in the second decade: a 17-year follow-up of Buffalo Creek survivors. J Am Acad Child Adolesc Psychiatry 1994; 33: 71-9.

8 Yule W, Bolton D, Udwin O, Boyle S, O'Ryan D, Nurrish J. The long-term psychological effects of a disaster experienced in adolescence: I: the incidence and course of PTSD. J Child Psychol Psychiatry 2000; 41: 503-11.

9 Bolton D, O'Ryan D, Udwin O, Boyle S, Yule W. The long-term psychological effects of a disaster experienced in adolescence: II: general psychopathology. $J$ Child Psychiat 2000; 41: 513-23.

10 Udwin O, Boyle S, Yule W, Bolton D, O'Ryan D. Risk factors for long-term psychological effects of a disaster experienced in adolescence: predictors of post traumatic stress disorder. J Child Psychiat 2000; 41: 969-79.

11 Terr LC. Chowchilla revisited: the effects of psychic trauma four years after a school-bus kidnapping. Am J Psychiatry 1983; 140: 1543-50.

12 Pfefferbaum B, Nixon SJ, Tucker P, Tivis RD, Moore VL, Gurwitch RH, et al Posttraumatic stress responses in bereaved children after the Oklahoma city bombing. J Am Acad Child AdolesC Psychiatry 1999; 38: 1372-9.

13 Nader K, Pynoos RS, Fairbanks L, Frederick C. Children's PTSD reactions one year after a sniper attack at their school. Am J Psychiatry 1990; 147: 1526-30.

14 Pynoos RS, Frederick C, Nader K, Arroyo W, Steinberg A, Eth S, et al. Life threat and posttraumatic stress in school-age children. Arch Gen Psychiatry 1987; 44: 1057-63.

15 Tyano S, Iancu I, Solomon Z, Sever J, Goldstein I, Touviana Y, et al. Sevenyear follow-up of child survivors of a bus-train collision. J Am Acad Child Adolesc Psychiatry 1996; 35: 365-73.

16 Winje $D$, Ukik A. Long-term outcome of trauma in children: the psychological consequences of a bus accident. J Child Psychol Psychiatry 1998; 39 : 635-42.

17 Morgan L, Scourfield J, Williams D, Jasper A, Lewis G. The Aberfan disaster: 33-year follow-up of survivors. Br J Psychol 2003; 182: 532-6.

18 Goenjian AK, Walling D, Steinberg AM, Karayan I, Najarian LM, Pynoos RS. A prospective study of posttraumatic stress and depressive reactions among treated and untreated adolescents 5 years after a catastrophic disaster. Am J Psychiatry 2005; 162: 2302-8.

19 Creamer M, Burgess P, McFarlane AC. Post-traumatic stress disorder: findings from the Australian National Survey of Mental Health and Well-being. Psychol Med 2001; 31: 1237-47.

20 Kessler A, Sonnega A, Bromet E, Hughes M, Nelson C. Posttraumatic stress disorder in the National Comorbidity Survey. Arch Gen Psychiatry 1995; 52: 1048-60.

21 McFarlane AC. Family functioning and overprotection following a natura disaster: the longitudinal effects of post-traumatic morbidity. Aust N Z J Psychiatry 1987; 21: 210-8. 
22 McFarlane AC. Posttraumatic phenomena in a longitudinal study of children following a natural disaster. J Am Acad Child Adolesc Psychiatry 1987; 26: 764-9.

23 McFarlane AC, Policansky SK, Irwin C. A longitudinal study of the psychological morbidity in children due to a natural disaster. Psychol Med 1987; 17: 727-38.

24 Australian Bureau of Statistics. Census of Population and Housing: SocioEconomic Indexes for Areas (SEIFA), Australia - Data Cube only. ABS, 2001.

25 Australian Bureau of Statistics. National Survey of Mental Health and Wellbeing of Adults: Users' Guide, 1997. Report No. 4327.0. ABS, 1999.

26 Australian Bureau of Statistics. Australian Standard Classification of Occupations (ASCO) (2nd edn). Report No. 1220.0. ABS, 1997.

27 American Psychiatric Association. Diagnostic and Statistical Manual of Mental Disorders (4th edn) (DSM-IV). APA, 1994.

28 World Health Organization. Composite International Diagnostic Interview (CIDI-AUTO). WHO, 1997

29 Goldney RD, Wilson D, Dal Grande E, Fisher LJ, McFarlane AC. Suicidal ideation in a random community sample: attributable risk due to depression and psychosocial and traumatic events. Aust N Z J Psychiatry 2000; 34: 98-106.

30 Weiss D, Marmar C. The impact of event scale-revised. In Assessing Psychological Trauma and PTSD (eds J Wilson, T Keane). Guilford Press, 1997.

31 Saunders JB, Aasland OG, Babor TF, de la Fuente JR, Grant M. Development of the Alcohol Use Disorders Identification Test (AUDIT): WHO collaborative project on early detection of persons with harmful alcohol consumption - II. Addiction 1993; 88: 791-804.

32 McFarlane A. The effects of stressful life events and disasters: research and theoretical issues. Aust N Z J Psychiatry 1985; 19: 409-21.
33 Andrews G, Henderson S, Hall W. Prevalence, comorbidity, disability and service utilisation. Overview of the Australian National Mental Health Survey. Br J Psychiatry 2001; 178: 145-53.

34 Green BL, Goodman LA, Krupnick JL, Corcoran CB, Petty RM, Stockton P, et al. Outcomes of single versus multiple trauma exposure in a screening sample. J Trauma Stress 2000; 13: 271-86.

35 Breslau N, Peterson EL, Schultz LR. A second look at prior trauma and the posttraumatic stress disorder effects of subsequent trauma: a prospective epidemiological study. Arch Gen Psychiatry 2008; 65: 431-7.

36 McFarlane A. Avoidance and intrusion in posttraumatic stress disorder. J NerV Ment Dis 1992; 180: 439-45.

37 Grayson DA, O'Toole BI, Marshall RP, Schureck RJ, Dobson M, Ffrench M, et al. Interviewer effects on epidemiologic diagnoses of posttraumatic stress disorder. Am J Epidemiol 1996; 144: 589-97.

38 Schulz KF, Chalmers I, Altman DG. The landscape and lexicon of blinding in randomized trials. Ann Intern Med 2002; 136: 254-9.

39 Erdman $\mathrm{HP}$, Klein $\mathrm{MH}$, Greist JH. Direct patient computer interviewing. J Consult Clin Psychol 1985; 53: 760-73.

40 World Health Organization. The ICD-10 Classification of Mental and Behavioural Disorders: Clinical Descriptions and Diagnostic Guidelines. WHO, 1992.

41 Peters L, Issakidis C, Slade T, Andrews G. Gender differences in the prevalence of DSM-IV and ICD-10 PTSD. Psychol Med 2005; 35: 1-9.

42 Peters L, Slade T, Andrews G. A comparison of ICD-10 and DSM-IV criteria for posttraumatic stress disorder. J Trauma Stress 1999; 12: 335-43.

43 Sayer NA, Spoont M, Nelson D. Veterans seeking disability benefits for posttraumatic stress disorder: who applies and the self-reported meaning of disability compensation. Soc Sci Med 2004; 58: 2133-43.

EXTRA 